\title{
El sistema de gestión ambiental bajo NTC-ISO 14001:2015 para una institución de educación superior desde la planificación y control operacional
}

\author{
The environmental management system under \\ NTC-ISO 14001:2015 for a higher education institution \\ from the operational planning and control
}
O sistema de gestão ambiental sob NTC-ISO 14001: 2015 para uma instituição de ensino superior desde a planificação e controle operacional

Recibido: 11 de diciembre de 2017 Revisado: 15 de enero de 2018 Aceptado: 24 de marzo de 2018

Yenith Cristina Ortiz Gonะále***

Universidad Santo Tomás

Julián Efraín Rincón Laverde ${ }^{* * *}$

Universidad Tecnológica de Pereira

Jorge Mario García Santa***

Universidad Santo Tomás

Mariela Gallo Montenegro ${ }^{* * * *}$

Universidad Santo Tomás

\footnotetext{
Artículo de resultado de investigación. DOI: http://dx.doi.org/10.15332/s2145-1389.2018.0001.07

** Ingeniera industrial. Especialista en Gerencia de Productividad y Calidad. Magíster en Calidad y Gestión Integral. Estudiante del doctorado en Educación. Docente investigadora. Correo electrónico: induscris4@gmail.com.

*** Administrador ambiental. Especialista en Administración y Gerencia de Sistemas de la Calidad. Magíster en Calidad y Gestión Integral. Correo electrónico: julian.rincon@utp.edu.co.

**** Administrador ambiental. Especialista en Gerencia en la Seguridad y Salud en el Trabajo. Magíster en Calidad y Gestión Integral. Docente Universidad Santo Tomás. Correo electrónico: jorgegarcias@usantotomas.edu.co ${ }^{* * * * *}$ Ingeniera de sistemas. Especialista en Docencia Universitaria. Especialista en Sistemas de la Calidad. Magíster en Calidad y Gestión Integral.
Docente Universidad Santo Tomás. Correo electrónico: marielagallo@usantotomas.edu.co
} 


\section{RESUMEN}

Este artículo tiene por objetivo identificar los principales cambios de la NTC-ISO 14001:2015 frente a la versión 2004 (la estructura de alto nivel, el concepto de ciclo de vida del producto, la comunicación externa de los impactos ambientales y el control de los cambios planificados del sistema), y proponer las actividades necesarias para dar cumplimiento a los cambios contenidos en el numeral 8.1, "Planificación y control operacional", a través de un plan de acción diseñado específicamente para el caso de una institución de educación superior. Para ello, se realizó un análisis comparativo entre las versiones 2015 y 2004 de la NTG-ISO 14001, el cual dio como resultado la identificación de los nuevos requisitos que deben cumplir las organizaciones que deseen mantener la certificación de su sistema de gestión ambiental. La segunda etapa consistió en definir a manera de alternativas las acciones que deben incluir en el plan de acción las organizaciones que deseen llevar a cabo la transición a la versión 2015 de la norma, con el fin de dar cumplimiento a los requisitos identificados en la etapa uno. Después de la revisión, se puede argumentar que sí existen cambios en los requisitos para la transición frente al numeral 4.4.6, "Control operacional", de la versión 2004, en aspectos como: el enfoque de ciclo de vida de productos y servicios; el control del proceso de diseño y desarrollo; la comunicación externa de posibles impactos significativos asociados a las actividades de transporte, entrega, uso y disposición final de productos y servicios; y el control de la planificación de los cambios que las organizaciones deben cumplir a través de un plan de transición con las actividades señaladas en este documento.

Palabras clave: control operacional, gestión ambiental, transición, ISO 14001:2015.

\section{ABSTRACT}

The purpose of this article is to identify the main changes of the NTC-ISO 140012015 compared to the 2004 version (the high-level structure, the concept of the product life cycle, the external communication of environmental impacts and the control of planned changes in the system), and propose the necessary activities to comply with the changes included in paragraph 8.1, "Operational planning and control", through an action plan designed specifically for the case of a higher education institution. To this end, a comparative analysis was made between the 2015 and 2004 versions of the NTC-ISO 14001, which resulted in the identification of the new requirements that organizations wishing to maintain the certification of their environmental management system must comply with. The second stage was to define as alternatives the actions that organizations that wish to carry out the transition to the 2015 version of the standard should include in the action plan, in order to comply with the requirements identified in the stage one. After the review, it can be argued that there are changes in the requirements for the transition compared to paragraph 4.4.6, "Operational control", of the 2004 version, in aspects such as: the approach of products and services life cycle; the control of the design and development process; the external communication of possible significant impacts associated with the activities of transport, delivery, use and final disposal of products and services, and the control in change planning, that the organizations must comply through a plan of transition with the activities indicated in this document.

Keywords: Operational control, environmental management, transition, ISO 14001:2015. 


\section{RESUMO}

Este artigo tem por objetivo identificar as principais alterações da NTC-ISO 14001: 2015 respeito a versão de 2004 (a estrutura de alto nível, o conceito de ciclo de vida do produto, a comunicação externa dos impactos ambientais e o controle das mudanças planejadas do sistema), e propor as atividades necessárias para 0 cumprimento das mudanças contidas no numeral 8.1, "planificação e controle operacional", através de um plano de ação criado especificamente para o caso de uma instituição de ensino superior. Para tanto, se realizou uma análise comparativa entre as versões 2015 e 2004 da NTC-ISO 14001, do qual resultou a identificação dos novos requisitos que devem cumprir as organizações que desejam manter a certificação de seu sistema de gestão ambiental. A segunda etapa consistiu em definir as alternativas e as ações que devem incluir no Plano de Ação as organizações que desejam realizar a transição para a versão 2015 da norma, a fim de cumprir os requisitos identificados na primeira etapa. Depois da revisão, pode-se argumentar que se existirem mudanças nos requisitos para a transição em relação ao numeral 4.4.6, "Controle operacional", da versão 2004, em aspectos como: 0 enfoque do ciclo de vida de produtos e serviços; o controle do processo de desenho e desenvolvimento; a comunicação externa dos possíveis impactos significativos associados às atividades de transporte, entrega, uso e disposição final de produtos e serviços, e o controle da planificação das mudanças, que as organizações devem cumprir por meio de um plano de transição com as atividades identificadas neste documento.

Palavras-chave: controle operacional, gestão ambiental, transição, ISO 14001:2015.

\section{INTRODUCCIÓN}

El concepto de gestión ambiental se aborda por primera vez a nivel mundial a principios de la década del 70, en la Cumbre de la Tierra celebrada en Estocolmo, donde se manifestó la preocupación por la problemática ambiental a nivel global. En 1992, posterior a la Cumbre de Río de Janeiro, la British Standard (BS) crea la norma de Gestión Ambiental BS 7750. Esta norma fue el modelo con el que la Organización Internacional de Estandarización (ISO) consideró la constitución del Comité Técnico ISO/TC 207 Gestión Ambiental, que fue encargado de elaborar y mantener las normas técnicas en el tema ambiental. Es así como la ISO elaboró en 1996 la primera versión de la norma internacional ISO 14001 con los requisitos para un sistema de gestión ambiental (Kleinová y Szaryszová, 2014).

Según Ortiz y Ramírez (2017), Colombia ha reportado un total de 2.983 certificaciones para el año 2015, representando una disminución del $13 \%$ comparado con el año 2014, donde se obtuvieron 3.433 certificados, y ha sido la más alta registrada en el país. Sin embargo, Colombia se mantiene como uno de los países con mayor número de certificados ISO 14001 en Centro y Suramérica (ISO, 2016).

Como resultado de las revisiones realizadas por el Comité ISO/TC 207, la NTC-ISO 14001 tuvo dos actualizaciones. En el 2004 incorporó el modelo del ciclo PHVA (sigla de planear, hacer, verificar y actuar) en la estructura de los sistemas de gestión ambiental; la identificación de aspectos ambientales en actividades, productos y servicios, y la necesidad de definir el alcance del sistema de gestión ambiental. La actualización del 2015 incorporó la estructura de alto nivel para hacerla fácilmente integrable con otros sistemas de gestión, el concepto de ciclo de vida del producto y el enfoque por procesos (Ortiz, 2016). 
La estructura de alto nivel está conformada por diez capítulos; en el capítulo 8, "Operación", se encuentra el numeral 8.1, "Planificación y control operacional" (ver figura 1). En esta estructura se establecen los lineamientos para que las organizaciones seleccionen el tipo de método de control en los procesos del sistema de gestión y puedan alcanzar los resultados establecidos y deseados (eficacia).

Este artículo identifica los cambios en la actualización de la NTC-ISO 14001:2015, específicamente para el requisito 8.1, "Planificación y control operacional de los Sistemas de Gestión Ambiental (SGA)", y define las acciones que debe ejecutar una institución de educación superior (IES), que en Colombia según la Ley 30 de 1992 son las universidades, instituciones técnicas profesionales e instituciones universitarias 0 escuelas tecnológicas, que cuentan con un reconocimiento del Ministerio de Educación Nacional (MEN), y sus actividades se basan en la formación académica en ocupaciones de carácter operativo e instrumental y de especialización en su respectivo campo de acción; o dedicadas a la investigación científica o tecnológica, producción, desarrollo y transmisión del conocimiento y de la cultura universal y nacional.

\section{METODOLOGÍA}

La metodología se estructuró en dos etapas. En primer lugar, se realizó un análisis comparativo entre las versiones 2015 y 2004 de la NTC-ISO 14001, el cual dio como resultado la identificación de los nuevos requisitos que deben cumplir las organizaciones que deseen mantener la certificación de su sistema de gestión ambiental (ver tabla 1).

La segunda etapa consistió en definir, a manera de alternativas, las acciones que deben incluir en el plan de acción las organizaciones que deseen llevar a cabo la transición a la versión 2015 de la norma, con el fin de dar cumplimiento a los requisitos identificados en la etapa uno.

Fiǵura 1. Estructura de alto nivel. NTC-ISO 14001:2015, capítulo 8.1

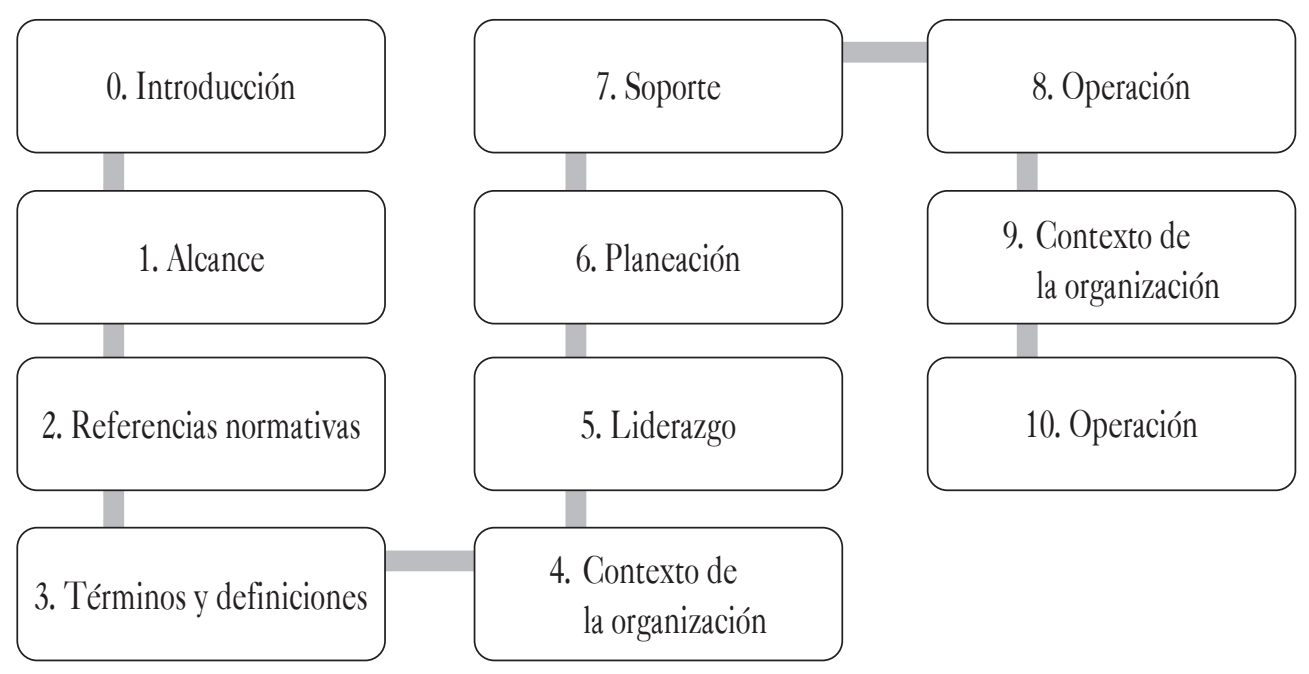

Fuente: elaboración propia basados en British Standards Institution (2016). 
Tabla 1. Cambios en los requisitos y las evidencias del numeral 8.1, "Planificación y control operacional"

\begin{tabular}{|c|c|c|c|}
\hline TEMA & REQUISITO & INTERPRETACIÓN & EVIDENCIA \\
\hline \multirow{4}{*}{$\begin{array}{l}\text { Planificación y control } \\
\text { operacional }\end{array}$} & \multirow{4}{*}{ 8.1 NTC-ISO 14001:2015 } & $\begin{array}{l}\text { La IES debe definir los controles frente } \\
\text { a los cambios que proyecte ejecutar en } \\
\text { el sistema de gestión ambiental o en la } \\
\text { organización, teniendo en cuenta los } \\
\text { posibles efectos negativos que puedan } \\
\text { generarse en el ambiente. }\end{array}$ & $\begin{array}{l}\text { El control y planificación de los } \\
\text { cambios, teniendo en cuenta sus } \\
\text { consecuencias y acciones para mitigar } \\
\text { los efectos adversos. }\end{array}$ \\
\hline & & \multicolumn{2}{|c|}{ PERSPECTIVA DEL CICLO DE VIDA } \\
\hline & & $\begin{array}{l}\text { La IES debe considerar el cumplimiento de } \\
\text { los requisitos ambientales en el proceso de } \\
\text { diseño y desarrollo para cada etapa del ciclo } \\
\text { de vida de los servicios. } \\
\text { Definir los requisitos ambientales para la } \\
\text { compra de productos y servicios. }\end{array}$ & $\begin{array}{l}\text { Los controles para asegurar que los } \\
\text { requisitos ambientales se incluyen en } \\
\text { el proceso de diseño y desarrollo del } \\
\text { producto o servicio, considerando cada } \\
\text { etapa del ciclo de vida. }\end{array}$ \\
\hline & & $\begin{array}{l}\text { La IES debe revisar si en la prestación de } \\
\text { los servicios de educación, investigación } \\
\text { y extensión, se realizan actividades de } \\
\text { transporte, entrega, uso, tratamiento y } \\
\text { disposición final de productos o servicios. } \\
\text { Posteriormente, se deben identificar } \\
\text { los aspectos e impactos ambientales } \\
\text { significativos que se puedan presentar } \\
\text { en estas actividades y, de acuerdo con el } \\
\text { resultado, tomar la decisión de comunicar } \\
\text { esta información a las partes interesadas } \\
\text { pertinentes. }\end{array}$ & $\begin{array}{l}\text { El registro de la revisión de la necesidad } \\
\text { de suministrar información acerca de } \\
\text { los impactos ambientales potenciales } \\
\text { significativos, asociados con el } \\
\text { transporte, entrega, uso, tratamiento } \\
\text { y disposición final de sus productos o } \\
\text { servicios. }\end{array}$ \\
\hline
\end{tabular}

Fuente: NTC-ISO 14001:2015 (Icontec, 2015).

\section{RESULTADOS Y DISCUSIÓN}

Como resultado del análisis realizado en la etapa uno de la metodología, se pudo identificar que, a pesar de que la estructura de alto nivel contenida en el Anexo SL (ISO, 2012) contempló de manera general el numeral 8.1 para todos los sistemas de gestión. En los sistemas de gestión ambiental este requisito quedó específico, lo que ha dificultado su integración con otros modelos. Para que los procesos operen bajo condiciones controladas, la organización debe identificar y valorar los aspectos e impactos ambientales de sus actividades, productos y servicios, y gestionar los de valor significativo. Seguidamente, tiene que definir las características, límites y rangos óptimos en los que deben operar los procesos que identificaron los aspectos ambientales significativos (criterios de operación), y definir los controles para asegurar que se cumplan los criterios de operación en los procesos (eliminación, sustitución, ingeniería, administrativos o combinados). Estos controles pueden documentarse en procedimientos, manuales, guías o instructivos, en la cantidad que la organización considere necesario y de acuerdo con la complejidad de las actividades y el nivel de competencia del personal (Icontec, 2015).

Uno de los elementos de mayor relevancia introducidos en la actualización de la NTC-ISO 14001:2015 es 
el enfoque del ciclo de vida del producto o servicio, el cual contempló los aspectos e impactos ambientales relacionados con las diferentes actividades asociadas a la elaboración del producto o prestación del servicio, como por ejemplo, la adquisición de las materias primas o insumos, el transporte de estas, el diseño, la producción, el almacenamiento del producto, la entrega del producto o prestación del servicio y la disposición final (Icontec, 2007a). Esto implicó que el alcance de los sistemas de gestión ambiental se ampliase, y que fuera necesario considerar y controlar las actividades realizadas por terceros (proveedores, contratistas y clientes) que están relacionadas con aspectos e impactos ambientales significativos.

Con la aplicación de este requisito se generó un reto para las organizaciones prestadoras de servicios educativos, teniendo en cuenta que en el ciclo de vida de los servicios no se ejecutan actividades de compra de materias primas, transporte, producción, entrega, tratamiento y disposición final; sino actividades como diseño, contratación y compra de materiales e insumos complementarios y la prestación del servicio (Icontec, 2007a).

Fiǵura 2. Sistema de producto para el análisis del ciclo de vida del producto

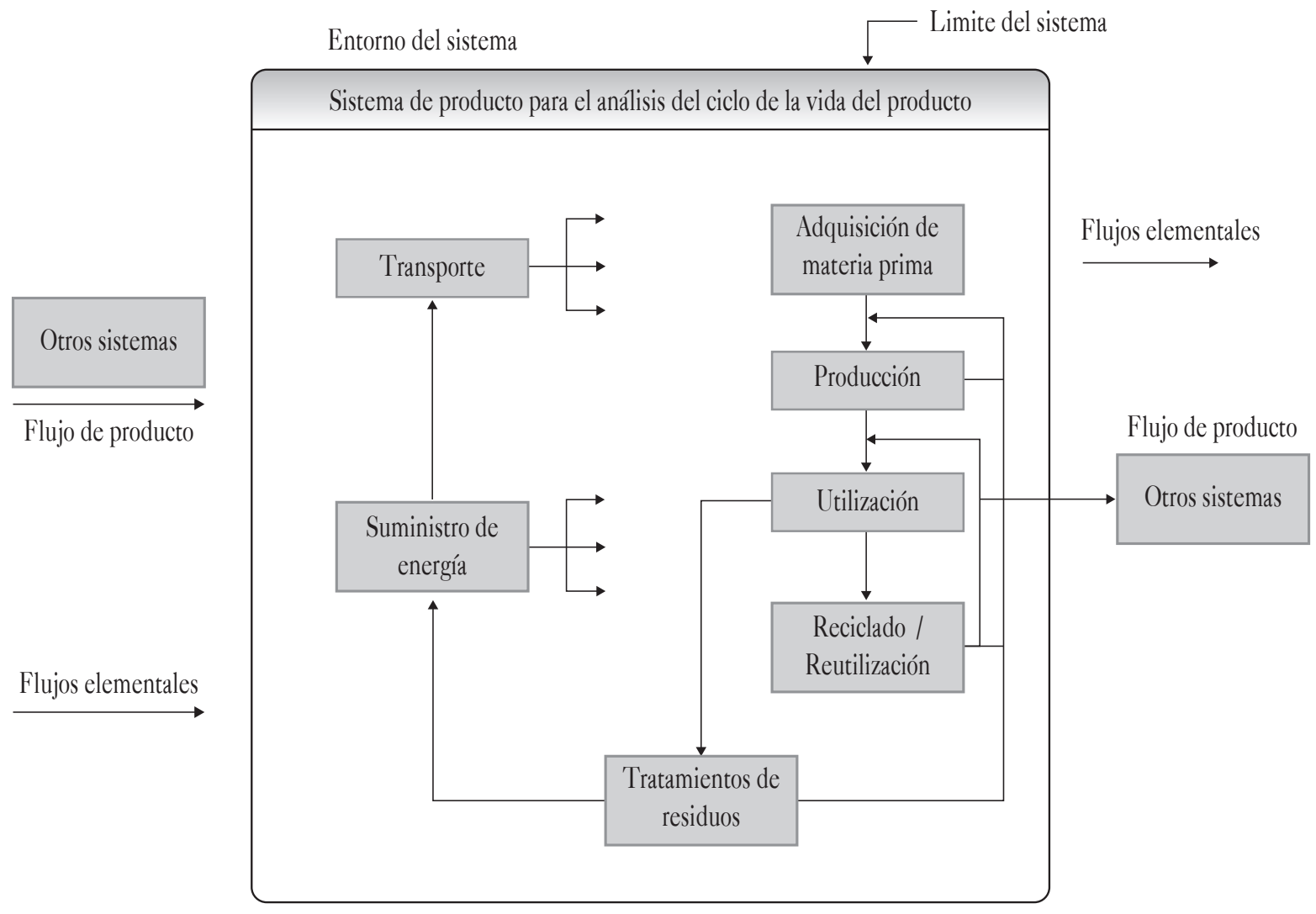

Fuente: NTC-ISO 14040. Gestión Ambiental. Análisis de ciclo de vida (Icontec, 2007a). 
Figura 3. Ciclo de vida del servicio

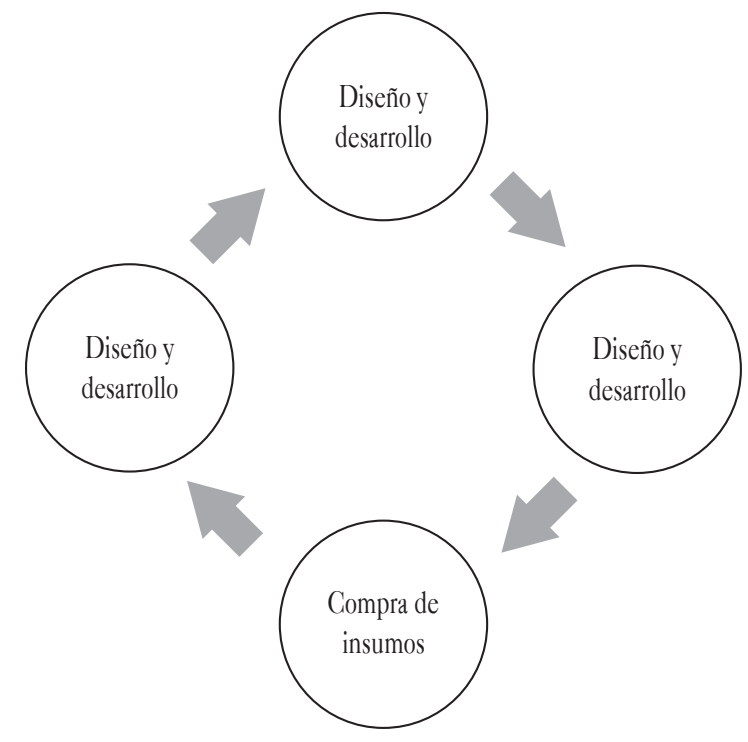

Fuente: elaboración propia.

La compra de materiales e insumos relacionados con las actividades administrativas y de aseo, ha sido una práctica frecuente en las empresas de servicios, y esto no es la excepción en las IES. En esta actividad se debe dar cumplimiento a lo establecido por la NTC-ISO 14001:2015, frente a la definición por parte de las organizaciones de los requisitos ambientales para la compra de insumos y materiales (Kleinová y Szaryszová, 2014). Las organizaciones pueden definir criterios ambientales de selección de proveedores que favorezcan aquellos que tengan al día toda la documentación legal (licencias ambientales, permisos de explotación, disposición en rellenos autorizados), suministren productos amigables con el ambiente (biodegradables, reciclables, reutilizables) y acepten compromisos de posconsumo, como la recuperación y responsabilidad frente a empaques, embalajes y disposición final de los productos (Passetti y Tenucci, 2016).

Todos los criterios, controles y disposiciones señalados, que el sistema de gestión ambiental debe establecer para la transición a NTC-ISO 14001:2015 y que están relacionados con el numeral 8.1, deben ser comunicados efectivamente a proveedores y contratistas. Razón por la que la organización debe incluir esta información en el plan de comunicación, considerando la necesidad de suministrar información acerca de los impactos ambientales potenciales significativos asociados con el transporte o la entrega, el uso, el tratamiento al fin de la vida útil y la disposición final de sus productos o servicios (Icontec, 2015).

Finalmente, la IES debe proyectar, planificar y controlar los cambios organizacionales en el sistema de gestión ambiental y en los productos, servicios e infraestructura que puedan estar asociados a aspectos e impactos ambientales significativos. Entre estos están los relacionados con las entradas al proceso de diseño y desarrollo de productos y servicios; así, por ejemplo, una modificación en este proceso puede generar aspectos ambientales como mayor consumo de insumos, energía, agua o incrementos en la generación de residuos, emisiones o disposiciones, que se asocian a impactos negativos en el ambiente (Icontec, 2015).

Los resultados de la etapa 2 están descritos a continuación:

Figura 4. Fases para la transición

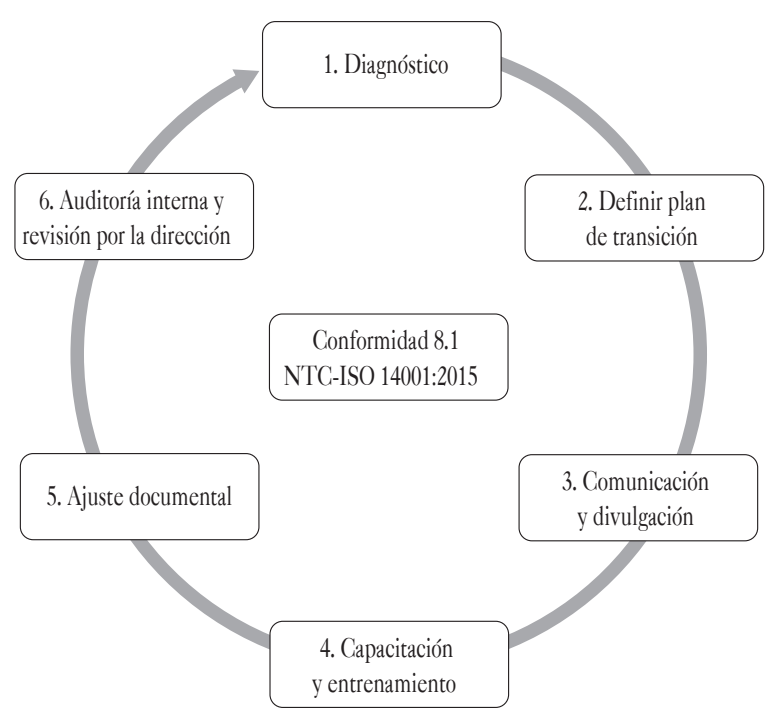

Fuente: elaboración propia. 
Como resultado de esta investigación, se propuso que las organizaciones educativas que planeen realizar la transición a la norma NTC-ISO 14001:2015, para dar cumplimiento con el requisito 8.1, deben realizar la siguiente secuencia de actividades.

El primer paso es realizar un diagnóstico que permita identificar las brechas del sistema de gestión ambiental frente a los requisitos del numeral, y formular a manera de pregunta la información de la tabla 1 , y de esta forma convertirla en una lista de chequeo que permita establecer el estado de cumplimiento del sistema de gestión ambiental frente a los requisitos de la versión 2015 (International Accreditation Forum, 2015).
El segundo paso propuesto es definir un plan de acción-transición, tomando como base el resultado del diagnóstico realizado con la lista de chequeo del paso anterior. El plan debe considerar las actividades, responsables y fechas para dar cumplimiento a los cambios en los requisitos de la versión 2015 (International Accreditation Forum, 2015).

Como parte del plan de transición, se tiene que considerar la definición de los criterios de operación y controles en los procesos con significancia en cuanto a los riesgos y oportunidades, requisitos legales y planificación de acciones, teniendo en cuenta que en la versión 2004 solo se consideraba el control operacional para los procesos con aspectos ambientales significativos.

Tabla 2. Plan de transición

\begin{tabular}{|c|c|c|}
\hline ACTIVIDAD & RESPONSABLE & FECHA \\
\hline $\begin{array}{l}\text { Definir los criterios de operación y los controles en los procesos con } \\
\text { significancia para: } \\
\text { - Riesgos y oportunidades. } \\
\text { - Requisitos legales. } \\
\text { - Planificación de acciones. } \\
\text { - Actividades del Análisis de Ciclo de Vida (ACV). }\end{array}$ & & \\
\hline $\begin{array}{l}\text { Revisar los planes de mantenimiento preventivo y correctivo de } \\
\text { maquinaria y equipos. }\end{array}$ & & \\
\hline $\begin{array}{l}\text { Controlar el cumplimiento de los requisitos ambientales en el proceso de } \\
\text { diseño y desarrollo para cada una de las etapas. }\end{array}$ & & \\
\hline Controlar el proceso de compras (revisar los requisitos ambientales). & & \\
\hline $\begin{array}{l}\text { Revisar la posibilidad que se presenten impactos ambientales significativos } \\
\text { potenciales, asociados con las actividades de transporte o entrega, uso, } \\
\text { tratamiento y disposición final de productos y servicios, y considerar } \\
\text { la necesidad de comunicar esta información a las partes interesadas } \\
\text { pertinentes. }\end{array}$ & & \\
\hline $\begin{array}{l}\text { Implementar controles para la ejecución de cambios planificados y a las } \\
\text { consecuencias de los cambios no previstos (contexto de la organización, } \\
\text { gestión del riesgo, proceso de diseño y desarrollo). }\end{array}$ & & \\
\hline
\end{tabular}

Fuente: elaboración propia. 
Para empresas prestadoras de servicios como las IES, los criterios de operación se definieron para los procesos administrativos o de apoyo; por ejemplo, aspectos ambientales asociados al consumo de papel, al uso de servicios públicos domiciliarios (consumo de agua potable y electricidad o vertimiento de aguas residuales) y generación de residuos sólidos. Igualmente, los criterios operacionales en el diseño de los programas académicos, en aspectos como el acondicionamiento de la infraestructura donde se presta el servicio de educación (iluminación y ventilación natural), la capacidad de carga o resiliencia del ambiente donde se presta el servicio educativo (puede ser fijo o estacional, por ejemplo, salidas de campo académicas). De igual forma, la operación de los laboratorios y talleres en las universidades en aspectos ambientales como vertimientos, emisiones y residuos peligrosos (RESPEL), en los que existen elementos a controlar como temperatura, $\mathrm{pH}$, turbidez, DBO, DQO, carga biológica, material particulado entre otros, y según aplique en el caso.

La revisión de los planes de mantenimiento preventivo y correctivo de maquinaria y equipos, que asegure el funcionamiento óptimo y evite posibles pérdidas o desperdicios en los procesos, es otro elemento que tiene que ser considerado en el plan de transición de la organización.

Asimismo, de acuerdo con el enfoque de ciclo de vida del producto o servicio, la organización debe incluir las siguientes actividades en el plan de transición:

a. Controlar el cumplimiento de los requisitos ambientales en el proceso de diseño y desarrollo para cada etapa del ciclo de vida del servicio educativo, implica identificar y controlar los aspectos e impactos ambientales asociados a las etapas de contratación y adquisición de insumos, diseño de programas académicos y prestación del servicio de educación superior (ciclo de vida del servicio). Se deben tener en cuenta aspectos como el perfil y la competencia en gestión ambiental del personal contratado, el tipo de materiales e insumos que se definen para la prestación del servicio (características de los tableros, marcadores, borradores, material académico, equipos de cómputo, etc.) y la metodología definida (presencial, virtual 0 semipresencial).

b. Controlar el proceso de compras, incluidos los criterios ambientales para la selección de proveedores (de tal manera que aquellos que cuenten con sistemas de gestión ambiental implementados tengan mejor calificación), oferta de insumos amigables con el ambiente (reciclables, reutilizables, biodegradables), cumplimiento legal (licencias y permisos de explotación y aprovechamiento, fichas de datos de seguridad) y planes de posconsumo que aseguren la responsabilidad en la disposición final del producto o empaques del mismo, cuando aplique.

c. La organización debe revisar la posibilidad de presentar impactos ambientales significativos potenciales asociados con las actividades de transporte o entrega, uso, tratamiento y disposición final de productos y servicios, considerando la necesidad de comunicar esta información a las partes interesadas pertinentes. Sin embargo, para IES y en general para las empresas de servicios, la posibilidad de presentar impactos ambientales es baja, debido a que estas actividades no se realizan y los impactos relacionados con la prestación del servicio ya fueron tratados en el proceso de diseño y desarrollo.

Para la revisión e implementación de controles asociados a la ejecución de cambios planificados y a las consecuencias de los cambios no previstos que puedan afectar la operación del sistema de gestión ambiental, se recomienda hacer uso de la información obtenida en el análisis del contexto interno y externo y la gestión del riesgo, con el fin de identificar y mitigar cualquier 
tipo de impacto que se pueda generar en la organización y su sistema de gestión.

El tercer paso para la transición es la comunicación y divulgación del plan de transición definido por la organización a las partes interesadas pertinentes. Esta información debe ser comunicada en el interior de la organización y a las partes interesadas pertinentes, con el fin de garantizar su efectividad, razón por la cual se debe elaborar un plan de comunicaciones para la transición del sistema de gestión ambiental a la versión 2015, el cual incluye la información que se debe comunicar (el qué), la frecuencia y fechas (cuándo), el receptor del mensaje (a quién) y el canal o medio (cómo) (Icontec, 2015).

El cuarto paso es la capacitación y el entrenamiento que deben recibir las partes interesadas pertinentes que aportan en la eficacia del sistema de gestión ambiental, por lo que la organización debe definir el plan de capacitación y entrenamiento para fortalecer la competencia del personal que va a dar cumplimiento a los cambios en los requisitos de la NTG-ISO 14001:2015 (IAF, 2015).

El quinto paso es el ajuste de los documentos del sistema de gestión ambiental, de acuerdo a la necesidad identificada por la organización con la ejecución de los pasos anteriores formulados en este documento.

El sexto y último paso es la realización del ciclo de auditorías internas frente a los requisitos de la NTC-ISO 14001:2015, la formulación y ejecución de acciones correctivas de acuerdo con los resultados de la auditoría interna y la realización de la revisión por la dirección. Una vez se cuente con la ejecución de estos pasos, se considera pertinente contactar al organismo evaluador de la conformidad para solicitar la ampliación de alcance del sistema de gestión ambiental a la versión 2015 de la NTC-ISO 14001 (IAF, 2015).

\section{CONCLUSIONES}

Después de la revisión del numeral 8.1, "Planificación y control operacional”, de la NTC-ISO 14001:2015, se puede argumentar que sí existen cambios en los requisitos para la transición frente al numeral 4.4.6, "Control operacional", de la versión 2004, en aspectos como: el enfoque de ciclo de vida de productos y servicios; el control del proceso de diseño y desarrollo; la comunicación externa de posibles impactos significativos asociados a las actividades de transporte, entrega, uso y disposición final de productos y servicios; y el control de la planificación de los cambios que las organizaciones deben cumplir a través de un plan de transición con las actividades señaladas en este documento.

La perspectiva del ciclo de vida de productos y servicios de la versión 2015 no es un requisito desconocido para las organizaciones a nivel internacional, dicha condición está contemplada en el numeral 4.4.6 de NTC-ISO 14004:2004, al igual que en la NTC-ISO 14044:2007 (Icontec, 2004, 2007b). Pero se puede señalar que esta perspectiva ha sido trabajada principalmente por empresas de productos, razón por la cual el esfuerzo en su aplicación puede ser mayor para organizaciones de servicios como las IES.

La implementación del plan de transición propuesto en este documento para las instituciones de educación superior (IES), a través de los seis pasos (diagnóstico con la aplicación de la lista de chequeo, definición de plan de acción con los resultados del diagnóstico, elaboración y ejecución del plan de comunicaciones para la transición del SGA, elaboración y ejecución del plan de capacitación y entrenamiento, y ajuste documental), permite alcanzar la conformidad con el numeral 8.1 de la NTC-ISO 14001:2015 y mejorar la capacidad de gestionar sus impactos ambientales en todas las etapas del ciclo de vida del servicio. 


\section{REFERENCIAS}

British Standards Institution. (2016). ISO 14001 hoja informativa, antecedente y actualización hacia la revisión 2015. Recuperado de https://www.bsigroup.com/LocalFiles/es-MX/ISO\%2014001/ ISO14001DS.pdf

IAF - International Accreditation Forum. (2015). IAF ID 10:2015. Documento Informativo de IAF. Directrices para la planificación de la transición hacia ISO 14001:2015 (1. ${ }^{a}$ ed.). Sydney: IAF. Recuperado de http://www.iaf.nu/articles/ Informative_Documents」32

Icontec - Instituto Colombiano de Normas Técnicas y Certificación. (2004). Norma Técnica Colombiana NTC-ISO 14004. Sistemas de gestión ambiental. Directrices generales sobre principios, sistemas y técnicas de apoyo (2. ${ }^{a}$ ed.). Bogotá: Icontec.

Icontec - Instituto Colombiano de Normas Técnicas y Certificación. (2007a). Norma Técnica Colombiana NTC-ISO 14040. Gestión Ambiental. Análisis de ciclo de vida. Principio y marco de referencia (1. ${ }^{\mathrm{a}}$ ed.). Bogotá: Icontec.

Icontec - Instituto Colombiano de Normas Técnicas y Certificación. (2007b). Norma Técnica Colombiana NTC-ISO 14044. Gestión ambiental. Análisis de ciclo de vida. Requisitos y directrices. Requisitos del ciclo de

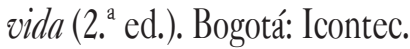

Icontec - Instituto Colombiano de Normas Técnicas y Certificación. (2015). Norma Ténnica Colombiana NTC-ISO 14001. Sistemas de gestión ambiental. Requisitos con orientación para su uso (2. ${ }^{2}$ ed.). Bogotá: Icontec.
ISO - International Organization for Standardization. (2012). Anexo SL. Recuperado de https://www. iso.org/sites/directives/2016/consolidated/index. xhtml

ISO - International Organization for Standardization. (2016). Evolution of ISO 14001 certificates in Colombia. Recuperado de http://www.iso.org/iso/ iso-survey

Kleinová, R., y Szaryszová, P. (2014). Comparison of new drafts of ISO 9001:2015 and ISO 14001:2015 standards in term of integration. Transfer inováciu, 29, 171-180. Retrieved from https://www.sjf.tuke. sk/transferinovacii/pages/archiv/transfer/29-2014/ pdf/171-180.pdf

Ortiz, Y. (2016). Curso de profundización opción de grado. Gestión ambiental y seguridad y salud ocupacional. Bogotá D. C.: Universidad Santo Tomás, Icontec.

Ortiz, Y., y Ramírez, M. (2017). Avances de las certificaciones ISO 9001 e ISO 14001 en Colombia. Revista Chilena de Economía y Sociedad, 11(1), 39-53. ISSN 0718-3933. Recuperado de https:// sitios.vtte.utem.cl/rches/wp-content/uploads/sites/8/2017/12/revista-chilena-economia-sociedadutem-vol11-n1-2017.pdf

Passetti, E., y Tenucci, A. (2016). Eco-efficiency measurement and the influence of organisational factors: Evidence from large Italian companies. Journal of Cleaner Production, 122, 228-239. http:// dx.doi.org/10.1016/j.jclepro.2016.02.035 